\title{
Diferentes Tipos de Reprodução Musical e sua Influência sobre o Tempo de Permanência dos Clientes em Restaurantes
}

\section{Different Types of Music Reproduction and their Influence on the Length of Stay of Customers in Restaurants}

\author{
Daniel Max de Sousa Oliveira ${ }^{1}$ \\ Elton Belz ${ }^{2}$ \\ Sylvio Ribeiro de Oliveira Santos ${ }^{3}$
}

\begin{abstract}
Resumo
Este estudo buscou ampliar o entendimento sobre a utilização da música em ambientes de consumo ao analisar o efeito de dois diferentes tipos de reprodução musical (ao vivo e mecanizada) sobre o tempo de permanência dos clientes em restaurantes. Por meio de um quase experimento foi observado que os clientes expostos à música ao vivo permaneciam por mais tempo no restaurante do que aqueles expostos à música mecanizada. Resultados adicionais ainda demonstraram que, na condição de música mecanizada, outros elementos do ambiente de consumo, como a quantidade de pessoas por mesa, desempenharam um efeito mais significativo sobre o tempo de permanência dos clientes no restaurante do que na condição de música ao vivo. Esses resultados oferecem uma contribuição aos estudos que analisam a manipulação da música em ambientes de consumo e seu efeito sobre o comportamento dos clientes.
\end{abstract}

Palavras-chave: música ao vivo; música mecanizada; tempo de permanência.

\begin{abstract}
This study sought to broaden the understanding of the use of music in consumption environments by analyzing the effect of two different types of musical reproduction (live and mechanized) on the length of time customers stay in restaurants. Through a quasi-experiment, it was observed that customers exposed to live music stayed longer in a restaurant than those exposed to mechanized music. Additional results also demonstrated that, in the condition of mechanized music, other elements of the consumption environment, such as the number of people per table, had a more significant effect on the length of stay of customers in the restaurant than in the condition of live music. These results contribute to studies that analyze the manipulation of music in consumption environments and its effect on customer behavior.
\end{abstract}

Keywords: live music; mechanized music; length of stay.

9 Mestre em Administração pela Universidade Federal de Santa Catarina (PPGA/UFSC). Doutorando em Administração pela Universidade Federal do Rio Grande do Sul (Escola de Administração/ UFRGS). Rio Grande do Sul (Escola de Administração/ UFRGS). 


\section{INTRODUÇÃO}

Um dos importantes avanços na área de comportamento do consumidor foi o reconhecimento de que as decisões dos clientes em ambientes de consumo podem ser influenciadas por elementos atmosféricos como a iluminação, a temperatura, o aroma e o som (BISWAS et al., 2017; BISWAS; SZOCS, 2019; KOTLER, 1973; MILLIMAN, 1986; SINHA; BAGCHI, 2019). A manipulação intencional desses elementos pode, por exemplo, estimular os clientes a permanecerem por mais tempo na loja (MILLIMAN, 1982), escolherem opções mais saudáveis (BISWAS et al., 2017; BISWAS; LUND; SZOCS, 2018) e reduzirem o ritmo com que se movimentam ou executam tarefas (MILLIMAN, 1986). Entre os elementos atmosféricos utilizados para criar um ambiente capaz de influenciar o comportamento do consumidor, a música tem se destacado, sobretudo em decorrência de sua fácil manipulação (BISWAS; LUND; SZOCS, 2018; CALDWELL; HIBBERT, 2002; MILLIMAN, 1986; TURLEY; MILLIMAN, 2000).

A partir de uma análise da literatura sobre a utilização da música em ambientes de varejo e serviço, foram encontrados estudos que manipulam o volume da música (BISWAS; LUND; SZOCS, 2018; SMITH; CURNOW, 1966; YALCH; SPANGENBERG, 1990), o ritmo da música (DONG; HUANG; LABROO, 2019; MILLIMAN, 1982; MILLIMAN, 1986), o gênero musical (MACIEL et al., 2010) e ainda a combinação entre duas ou mais dessas manipulações (HARRINGTON; OTTENBACHER; TREUTER, 2015).

No entanto Costa e Farias (2016) apresentaram uma possibilidade adicional de manipulação da música, que ainda não havia sido analisada em estudos anteriores, ao propor que a forma como a música é reproduzida no ambiente de consumo, ao vivo ou mecanizada, também pode influenciar o comportamento dos consumidores. Especificamente, Costa e Farias (2016) analisaram a utilização desses dois tipos de reprodução musical em supermercados e verificaram que os clientes expostos à música ao vivo se diziam predispostos a permanecer por um maior período de tempo no supermercado do que aqueles clientes expostos à música mecanizada. Aqui cabe ressaltar que o termo música mecanizada se refere à música reproduzida por meio de dispositivos eletrônicos, como o sistema de som de um restaurante. Por outro lado, música ao vivo diz respeito aquela que é ouvida de forma simultânea a sua execução por um(a) cantor(a) ou banda.

Apesar da contribuição oferecida pelo estudo de Costa e Farias (2016), torna-se pertinente utilizar uma abordagem em que o efeito dos diferentes tipos de reprodução musical sobre o tempo de permanência dos clientes em um ambiente de consumo seja mensurado de forma mais objetiva, uma vez que, no estudo mencionado, os clientes, abordados no interior dos supermercados, apenas indicaram, numa escala de concordância de 7 pontos, sua intenção de permanecer por mais tempo no estabelecimento.

O presente estudo contribui para ampliar o entendimento sobre essa questão ao analisar o efeito de dois diferentes tipos de reprodução musical sobre o tempo de permanência dos clientes por meio de observações diretas do horário de entrada e saída dos clientes em restaurantes. Por meio de um quase-experimento, foi observado que os clientes expostos à música ao vivo permaneciam por mais tempo no estabelecimento do que àqueles expostos à música mecanizada. Resultados adicionais demonstraram ainda que, na condição de música mecanizada (vs. ao vivo), outros aspectos do ambiente, como a quantidade de pessoas por mesa, exerciam uma influência mais significativa sobre o tempo de permanência dos clientes no restaurante. Esse resultado indica que, em ambiente com música mecanizada, a influência de outras variáveis do ambiente de consumo é mais determinante sobre o comportamento do consumidor.

Portanto, ao demonstrar que a reprodução da música ao vivo contribui para que os clientes permaneçam por mais tempo em restaurantes e, adicionalmente, evidenciar que em ambientes com música mecanizada outras variáveis atmosféricas têm maior oportunidade de influenciar nas ações dos clientes, o presente estudo oferece uma contribuição para a área de comportamento do consumidor, especificamente para aqueles estudos que analisam o papel da música como um elemento atmosférico em ambientes de varejo e serviço (BISWAS; LUND; SZOCS, 2018; CALDWELL; HIBBERT, 2002; DONG; HUANG; LABROO, 2019; MILLIMAN, 1986; TURLEY; MILLIMAN, 2000).

\section{FUNDAMENTAÇÃO TEÓRICA}

Nesta seção foram apresentados, inicialmente, os principais achados da literatura que analisa, como a manipulação intencional de elementos da música, como o volume e o ritmo, impactam no tempo de permanência dos clientes em ambientes de varejo e serviço. Em seguida, foi destacada uma discussão sobre os dois diferentes tipos de reprodução musical analisados neste estudo - mecanizada e ao vivo -, acompanhada das hipóteses de pesquisa.

\subsection{A influência da música sobre o tempo de permanência dos clientes}

Conforme destacaram Spangenberg e Yalch (2000), o tempo de permanência dos consumidores no interior das lojas representa um importante fator no varejo, uma vez que os varejistas acreditam que há uma relação direta entre o tempo despendido nas compras e a quantidade de itens adquiridos. Orientados por essa premissa, alguns estudos buscaram verificar se características específicas da música, manipuladas de maneira intencional em ambientes de 
varejo e serviço, poderiam influenciar no tempo de permanência dos clientes no interior desses ambientes de consumo (CALDWELL; HIBBERT, 2002; MILLIMAN, 1982; MILLIMAN, 1986; SMITH; CURNOW, 1966).

Smith e Curnow (1966), por exemplo, em um dos primeiros estudos da área de comportamento do consumidor a utilizar um design experimental com condições razoavelmente controladas, manipularam o volume da música, em moderadamente alto e moderadamente baixo, em dois supermercados. Como resultado, encontraram que a receita de vendas por minuto era maior na condição de música com volume alto. No entanto, esse resultado não ocorreu em decorrência do aumento absoluto nas vendas, mas foi motivado pelo menor tempo de permanência dos clientes no interior dos supermercados.

Por sua vez, Milliman (1982) destacou que havia evidência suficiente para se afirmar que o ritmo da música poderia afetar o fluxo dos consumidores dentro do ambiente de varejo. Especificamente, a movimentação dos compradores no interior de um supermercado era mais lenta quando eles estavam expostos a uma música com ritmo mais lento. Embora não mencionado diretamente pelo autor, acredita-se que aqueles clientes que se movimentavam mais lentamente tendiam a permanecer por um maior período de tempo no interior do supermercado.

De maneira similar aos estudos desenvolvidos no varejo, Milliman (1986) demonstrou que a música também poderia ser utilizada em ambientes de serviço, como os restaurantes. $O$ autor encontrou que, uma vez a comida servida, os clientes expostos à trechos de músicas com ritmo lento despendiam mais tempo para finalizar suas refeições comparados com aqueles que ouviam músicas com ritmo mais rápido. $O$ autor justificou esse resultado argumentando que o ritmo lento da música poderia contribuir para um ambiente mais calmo e relaxante, o que produzia níveis mais baixos de excitação nos clientes.

Em conformidade com o estudo de Milliman (1986), Caldwell e Hibbert (2002) demonstraram que os clientes expostos à música lenta, em média, despendiam quinze minutos a mais para finalizar suas refeições no restaurante do que aqueles sob a condição de música rápida. Os resultados desses dois estudos sugerem que há uma relação diretamente proporcional entre o ritmo da música e a dinâmica dos clientes no restaurante.

Portanto, algumas características da música podem ser manipuladas em ambientes de varejo e serviço no intuito de contribuir para uma permanência mais prolongada dos clientes. Os estudos, em sua maioria, indicam que músicas com volume mais baixo e ritmo mais lento despertam menos excitação nos consumidores e, consequentemente, contribuem para que eles desempenhem as atividades de maneira mais compassada, o que, em última instância, prolonga a sua permanência no interior dos ambientes de consumo (CALDWELL; HIBBERT, 2002).

Por outro lado, músicas com volume mais alto e ritmo mais rápido podem intensificar o sentimento de excitação dos consumidores, o que contribui para que eles executem as atividades rapidamente (CALDWELL; HIBBERT, 2002). Essa segunda condição pode ser relevante para restaurantes que privilegiam a rotação de clientes nas mesas como uma forma de aumentar as receitas (Milliman, 1986) e supermercados, que buscam escoar o fluxo de pessoas de forma mais rápida, reduzindo os efeitos negativos decorrentes da lotação (crowding).

Apesar das contribuições oferecidas pelos estudos citados anteriormente, para permitir a formulação da hipótese sobre a relação entre o tipo de reprodução musical (ao vivo ou mecanizada) e o tempo de permanência dos clientes no restaurante, recorreu-se a literatura que analisa os diferentes tipos de reprodução musical em outros contextos não associados diretamente ao consumo, pois na área de comportamento do consumidor, com exceção do estudo de Costa e Farias (2016), não há referência sobre essa relação.

\subsection{Música ao vivo e música mecanizada}

Embora a tecnologia tenha fornecido um método conveniente e de baixo custo para ouvir música, muitas pessoas continuam a optar por apresentações ao vivo. Mais do que isso, as experiências musicais mais significativas geralmente acontecem em eventos ao vivo. Dois fatores contribuem para essa preferência: primeiro, as pessoas gostam da conexão social que decorre de experimentar a música coletivamente; segundo, as pessoas gostam da sensação de estarem conectadas aos músicos, uma vez que cada apresentação ao vivo é diferente, de forma que os eventos se desenrolam de forma orgânica e imprevisível, ao contrário do que ocorre quando se ouve uma gravação, em que não há possibilidade de o público afetar diretamente no que o artista já criou (SWARBRICK et al., 2019).

Ainda nesse sentido, alguns autores asseguram que a música ao vivo é capaz de envolver, seduzir e mobilizar os ouvintes de maneira mais significativa do que a música reproduzida por meio de dispositivos eletrônicos (FREDERICKSON, 1989; FRITH, 2013; HERSCHMANN, 2008; IAZZETA, 1997). De acordo com lazzeta (1997), isso ocorre porque o ouvinte cria uma conexão com os elementos da produção sonora ao vivo, como o cantor e os instrumentos musicais, o que permite, inclusive, uma participação indireta nessa produção ao reconstruir internamente as sequências de notas executadas.

Semelhante ao que disse Swarbrick et al., (2019), Herschmann (2008) defende que a presença física do músico e dos instrumentos musicais representa outro aspecto que torna a performance ao vivo mais envolvente. A realidade física e social decorrente dessa presença torna a performance musical mais real e significativa, o que contribui para atrair as pessoas e torná-las mais envolvidas com aquela situação (HERSCHMANN, 2008). Portanto, a performance musical ao vivo, similar aos efeitos provocados pelo ritmo lento (MILLIMAN, 1986; CALDWELL; HIBBERT, 2002) e 
volume baixo (SMITH; CURNOW, 1966) poderia contribuir para manter os clientes no ambiente de consumo por um período de tempo mais prolongado.

No estudo de Costa e Farias (2016), de forma a complementar as considerações destacadas acima, foi demonstrado que os compradores expostos à música ao vivo se diziam propensos a permanecer durante um maior período de tempo no interior do supermercado do que aqueles expostos à música mecanizada. Diante desses argumentos, propõe-se a seguinte hipótese:

$\mathrm{H}_{1}$ : Os clientes expostos à música ao vivo permanecerão por mais tempo no restaurante do que aqueles expostos à música mecanizada.

Como destacado anteriormente, os autores que analisam os diferentes tipos de reprodução musical têm demonstrado que a música ao vivo é mais envolvente e consegue influenciar o indivíduo de maneira mais significativa (FREDERICKSON, 1989; FRITH, 2013; HERSCHMANN, 2008; IAZZETA, 1997). Propõe-se então que, em um ambiente com música ao vivo, a influência de outros fatores sobre o comportamento dos indivíduos é reduzida em decorrência da intensidade da influência exercida pela música. Contrariamente, espera-se que em um ambiente onde a música é reproduzida de forma mecanizada, outros fatores, além da música, exerçam influência mais significativa sobre o comportamento do consumidor.

Para testar essa hipótese, será verificada a influência que a quantidade de pessoas por mesa exercerá sobre o tempo de permanência dos clientes no restaurante nas duas condições de reprodução musical. Se a hipótese for confirmada, na condição de música mecanizada, a quantidade de pessoas por mesa deve influenciar de forma mais significativa o tempo de permanência dos clientes no restaurante, uma vez que, nessa condição, pelo fato da música ser menos envolvente, outros fatores do ambiente se tornam mais importantes e contribuem de forma decisiva sobre o tempo de permanência. É importante ressaltar que a utilização da variável quantidade de pessoas por mesa se deve tão somente à facilidade na sua coleta.

$\mathrm{H}_{2}$ : Na condição de música mecanizada, a quantidade de pessoas por mesa terá um efeito mais significativo sobre o tempo de permanência dos clientes no restaurante do que na condição de música ao vivo.

\section{MÉTODO}

Para analisar o efeito dos diferentes tipos de reprodução musical sobre o tempo de permanência dos clientes no restaurante, foi realizado um estudo com design quaseexperimental entre grupos (between subjects) com duas condições: música ao vivo e música mecanizada. Esses estudos são utilizados quando o pesquisador não detém controle sobre o processo de aleatoriedade dos participantes nas condições experimentais e não pode garantir que os participantes serão expostos à variável independente da mesma forma, algo que geralmente ocorre em experimentos de campo (HERNANDEZ; BASSO; BRANDÃO, 2014).

As observações e dados referentes às duas condições de reprodução musical foram coletados em restaurantes distintos. Assim, os dados referentes à condição de música mecanizada foram coletados no restaurante $A$, enquanto os dados referentes à música ao vivo foram coletados no restaurante $\mathrm{B}$. Ambos os restaurantes estão localizados na cidade de Pomerode, Santa Catarina, são especializados na culinária típica alemã e possuem como público-alvo, além dos moradores da cidade, turistas de várias localidades do Brasil. Em conformidade com o público-alvo, a música executada em ambos os restaurantes, por meio da utilização do sistema sonoro no restaurante A e por meio de uma banda no restaurante $B$, é tipicamente alemã.

O tipo de serviço oferecido em ambos os restaurantes é no formato buffet livre, em que os adultos pagam um valor único e podem se servir à vontade. Esse formato de serviço facilitou a coleta dos dados, pois não houve interferência do tempo de preparo do pedido sobre o tempo de permanência dos clientes no restaurante. As similaridades entre os restaurantes $A$ e $B$ indicam que os resultados encontrados se devem às manipulações experimentais da música e não às especificidades de cada estabelecimento.

Todas as observações e dados foram coletados aos domingos. O domingo foi escolhido por duas razões: primeiramente, esse é o dia da semana em que há um maior fluxo de pessoas nos restaurantes analisados. Além disso, aos domingos, as pessoas geralmente não estão pressionadas por restrições de tempo, o que é fundamental para os objetivos deste estudo.

O tempo de permanência foi registrado por meio de observações diretas do horário de entrada e saída do grupo de clientes. Para permitir um maior controle, os dados referentes à entrada dos clientes no restaurante foram coletados no período das $11 \mathrm{~h} 30$ às $13 \mathrm{~h} 30$. De acordo com os proprietários dos restaurantes analisados, a maior parte dos clientes costuma chegar dentro desse intervalo de tempo. Dessa forma, todos os grupos de clientes que entraram no restaurante durante esse intervalo de tempo fizeram parte da amostra do estudo. Por outro lado, não havia 
restrição de tempo quanto ao horário de saída dos clientes, ou seja, se o grupo de clientes chegasse ao restaurante dentro do intervalo de tempo estabelecido (das $11 \mathrm{~h} 30$ às 13h30), ele faria parte da amostra, independentemente do seu horário de saída.

Especificamente, o horário de entrada foi registrado após a entrada do grupo de clientes e o horário de saída foi registrado após todos os clientes de uma determinada mesa se levantarem em direção ao caixa para efetuar o pagamento. Essas e outras observações foram realizadas por observadores localizados em posições estratégicas nos restaurantes.

Além do tempo de permanência dos clientes, foi registrado o número de clientes por mesa para permitir análises adicionais. Após dois domingos consecutivos de coleta de dados, foram coletados dados de entrada e saída de clientes de 193 mesas, das quais 188 foram consideradas válidas (98 mesas na condição de música mecanizada e 90 na condição de música ao vivo), utilizadas na fase de análise. Afim de assegurar que os efeitos observados seriam, de fato, decorrentes das diferentes manipulações da música, outros elementos do ambiente, como iluminação, aroma e abordagem dos funcionários, foram mantidos constantes dentro da medida do possível.

\section{RESULTADOS}

Antes de apresentar os resultados referentes às hipóteses propostas, cabe destacar algumas estatísticas descritivas. No ambiente com música ao vivo, o tempo mínimo de permanência dos clientes foi de 22 minutos, enquanto o tempo máximo foi de 98 minutos. Ainda neste cenário, na mesa com o menor número de pessoas haviam 2 clientes, enquanto na mesa com o maior número de pessoas haviam 8 clientes. A quantidade média de clientes por mesa no ambiente com música ao vivo foi de 3,5 pessoas. Por sua vez, no ambiente com música mecanizada, o tempo mínimo de permanência dos clientes foi de 16 minutos, enquanto o tempo máximo foi de 79 minutos. Neste cenário, a mesa com o menor número de pessoas tinha 1 cliente, enquanto na mesa com o maior número de pessoas haviam 9 clientes. No ambiente com música mecanizada a quantidade média de pessoas por mesa era de 2,9 clientes.

Em relação às hipóteses, foi verificado que existe uma diferença no tempo médio de permanência (em minutos) dos clientes no restaurante entre as duas condições de reprodução musical $\left(M_{\text {aovivo }}=57,31 \mathrm{DP}=16,15\right.$ vs. $M_{\text {mecanizada }}$ $=48,11 \mathrm{DP}=14,14)$. Especificamente, observou-se que, na condição de música ao vivo, os clientes demoram, em média, nove minutos a mais para finalizar suas refeições e deixar o restaurante quando comparados aos clientes na condição de música mecanizada $(t(188)=-4,162 ; p<0,01)$. Dessa forma, a $\mathrm{H} 1$ foi confirmada.

Além dessa diferença, os resultados demonstraram que a influência da quantidade de clientes por mesa sobre o tempo de permanência dos clientes de cada mesa no restaurante varia de acordo com os diferentes tipos de reprodução musical. Para demonstrar esse efeito moderador exercido pela música, foi realizada uma análise de regressão linear múltipla com dois modelos. No modelo 1 , a quantidade de clientes por mesa (x), o tipo de reprodução musical (codificado como dummy variable onde $0=$ música mecanizada e $1=$ música ao vivo) (z) e o produto entre essas duas variáveis $(\mathrm{xz})$ foram inseridos como variáveis independentes, e o tempo de permanência dos clientes de cada mesa no restaurante (y) foi utilizado no modelo como variável dependente. No modelo 2 , o mesmo procedimento foi adotado, mas com uma exceção: o tipo de reprodução musical foi codificado de forma reversa, de modo que $0=$ música ao vivo e 1= música mecanizada.

As equações de regressão obtidas, respectivamente, a partir do modelo 1 e 2 foram as seguintes: (modelo 1): $y=49,65+5,27 x+6,75 z-2,42 x z$; (modelo 2): $y=56,40+2,86 x-6,75 z+2,42 x z$. O teste $t$ do coeficiente de regressão para o produto das duas variáveis independentes $(x z)$ foi, ainda que de forma marginal, estatisticamente significativo $(t=-1,749 ; p=0,08)$, o que sugere a presença de uma interação entre a quantidade de pessoas por mesa e o tipo de reprodução musical no que se refere ao efeito sobre o tempo de permanência.

De maior interesse para este estudo, foi verificado que o coeficiente não padronizado associado à variável $\mathrm{x}, \mathrm{o}$ qual indica o efeito da quantidade de clientes por mesa sobre o tempo de permanência daqueles clientes no restaurante quando a variável tipo de reprodução musical é igual a 0 , é maior no modelo $1(\beta=5,27 ; E P=1,067 ; t(188)=4,954$; $p<0,001 ; I C_{95 \%}: 3,180$ a 7,391$)$ do que no modelo $2\left(\beta=2,86 \mathrm{EP}=0,882 ; t(188)=3,246 ; p=0,001 ; I C_{95 \%}: 1,123\right.$ a $4,604)$. Lembrando que, no modelo 1 , quando o tipo de reprodução musical é igual a 0 , a análise se refere ao grupo de clientes expostos à música mecanizada. Contrariamente, no modelo 2 , quando o tipo de reprodução musical é igual a 0 , a análise se refere ao grupo de clientes expostos à música ao vivo. Pode-se afirmar, dessa forma, que o efeito da quantidade de pessoas por mesa sobre o tempo de permanência dos clientes no restaurante é mais significativo na condição de música mecanizada, pois, para cada cliente adicional na mesa, o grupo de clientes expostos à música mecanizada permanecerá 5,27 minutos a mais no restaurante, enquanto que, na condição de música ao vivo, eles permanecerão apenas por 2,86 minutos adicionais (ver tabela 1). Esse resultado oferece suporte à $\mathrm{H}_{2}$ 
Tabela 1 - Resultados da regressão linear múltipla

\begin{tabular}{|l|c|c|c|c|}
\hline & \multicolumn{2}{|c|}{$\mathbf{1}^{\mathbf{0}}$ modelo } & \multicolumn{2}{c|}{$\mathbf{2}^{\mathbf{0}}$ modelo } \\
\hline Variáveis independentes & $\boldsymbol{\beta}^{\mathbf{1}}$ & $\boldsymbol{p}$-value & $\boldsymbol{\beta}^{1}$ & $\boldsymbol{p}$-value \\
\hline Intercepto & 49,65 & 0,000 & 56,40 & 0,000 \\
\hline Quantidade de pessoas/mesa (x) & 5,27 & 0,000 & 2,86 & 0,001 \\
\hline Tipo de reprodução musical (z) & 6,75 & 0,001 & $-6,75$ & 0,001 \\
\hline $\mathbf{X z}$ & $-2,42$ & 0,082 & 2,42 & 0,082 \\
\hline $\mathbf{R}^{2}$ & 0,232 & & 0,232 & \\
\hline
\end{tabular}

${ }^{1}$ Coefientes não padronizados

Fonte: elaborado pelos autores.

\section{ANÁLISE DOS RESULTADOS}

Os resultados demonstraram que os clientes permanecem por um maior período de tempo no restaurante quando estão expostos à música ao vivo. Baseado nas concepções de lazzeta (1997), esse comportamento se deve ao fato de que a música ao vivo é capaz de envolver os ouvintes de maneira mais significativa do que a música reproduzida por meio de dispositivos eletrônicos, contribuindo, assim, para tornar o ambiente mais agradável e atrativo. Além disso, conforme proposto por Herschman (2008), a realidade física e social da reprodução ao vivo pode ter intensificado a influência da música sobre os clientes.

Esse efeito produzido pela música ao vivo sobre o tempo de permanência dos clientes é semelhante àquele provocado pela música com ritmo lento (CALDWELL; HIBBERT, 2002; MILLIMAN, 1986) ou com volume baixo (SMITH; CURNOW, 1966). No entanto, o mecanismo que explica os efeitos produzidos por essas diferentes formas de manipulação da música sobre o tempo de permanência dos clientes não aparenta ser o mesmo. Por um lado, os estudos que demonstram que a música com ritmo lento ou volume moderadamente baixo contribui para um tempo de permanência mais prolongado nos ambientes de consumo atribuem esse efeito às propriedades de excitação da música.

De acordo com Berlyne (1971), a música com baixas propriedades de excitação, como aquela com ritmo lento ou volume baixo, contribui para que os indivíduos desempenhem as atividades mais lentamente. Milliman (1986) complementa essa explicação ao destacar que a música com baixa excitação produz um ambiente mais calmo e relaxante, o que contribui para que os clientes percam a noção do tempo, prolongando, assim, sua permanência no restaurante.

Por outro lado, as propriedades de excitação não representam o mecanismo mais apropriado para explicar o efeito da música ao vivo sobre o tempo de permanência dos clientes no restaurante. Uma explicação mais provável para esse efeito pode estar associada aos processos emocionais ou afetivos dos indivíduos (DONOVAN; ROSSITER, 1982), de tal modo que, na condição de música ao vivo, o maior tempo de permanência pode ser uma resposta afetiva dos clientes ao ambiente, que se mostra mais agradável e atrativo.

Os resultados também demonstraram que, na condição de música mecanizada, a quantidade de pessoas por mesa influenciou de forma mais significativa o tempo de permanência do grupo de clientes no restaurante. Propõese que, por meio da reprodução mecanizada, a música torna-se um elemento menos atrativo para os clientes no ambiente (FRITH, 2013). Assim, eles tendem a valorizar mais ou priorizar elementos adicionais, como a interação com as demais pessoas do grupo. Uma vez que a interação assume um papel importante durante a experiência no restaurante, a quantidade de pessoas pode contribuir de maneira mais decisiva no tempo de permanência do grupo. Por outro lado, na condição de reprodução ao vivo, como a música é um elemento mais atrativo (FRITH, 2013), ela tende a ser mais valorizada ou priorizada pelos clientes, o que diminui a oportunidade para que outros elementos do ambiente, ou mesmo a interação do grupo, exerça uma influência significativa sobre o tempo de permanência.

\section{CONSIDERAÇÕES FINAIS}

Neste estudo, em que se buscou analisar os efeitos provocados por dois diferentes tipos de reprodução musical (ao vivo ou mecanizada) sobre o comportamento do consumidor, foi verificado que a música ao vivo contribui para que os clientes permaneçam por um maior período de tempo em restaurantes. Por meio desse resultado, este estudo amplia o entendimento apresentado por Costa e Farias (2016) de que, ao serem expostos à música ao vivo, os clientes se diziam predispostos a permanecer por mais tempo no supermercado, demonstrando, por meio de observações diretas do horário de entrada e saída dos clientes no restaurante, que esse efeito realmente ocorre.

Resultados adicionais ainda demonstraram que outros fatores, como a quantidade de pessoas por mesa, influenciam no tempo de permanência dos clientes de forma mais significativa na condição de música mecanizada. Esse resultado indica que a música mecanizada, por ser menos atrativa, não preenche a experiência de consumo por 
completo, o que abre oportunidades para que fatores adicionais, como a interação com as demais pessoas na mesa, influenciem de forma mais decisiva o comportamento do consumidor. Contrariamente, a música ao vivo é um elemento com presença mais marcante na experiência, diminuindo, assim, prováveis lacunas que possam ser preenchidas por outros estímulos externos. Esses resultados indicam que a utilização de música mecanizada pode ser mais apropriada para a realização de estratégias conjuntas com outros estímulos atmosféricos, como a iluminação ou aroma.

Adicionalmente, a concepção de que os clientes permanecem por mais tempo no restaurante quando estão expostos à música ao vivo também está associada a algumas implicações gerenciais. Em determinados tipos de restaurantes, como bares e cafeterias, onde o maior tempo de permanência dos clientes pode influenciar diretamente na quantidade consumida por eles e, consequentemente, nas receitas da empresa, a utilização de música ao vivo pode ser uma interessante ferramenta estratégica. Por outro lado, em restaurantes, bares e outros estabelecimentos de consumo que privilegiam um menor tempo de permanência dos clientes no ambiente, como os fast-foods, a música mecanizada ainda parece ser mais adequada. Cabe destacar que a utilização da música ao vivo está associada ao custo adicional de contratação da atração musical. Assim, a organização que optar por utilizar música ao vivo como um instrumento para tornar a permanência dos clientes mais prolongada deve avaliar se os benefícios proporcionados por essa estratégia serão superiores aos custos necessários para sua implementação.

Apesar das contribuições oferecidas, a generalização dos resultados encontrados neste estudo deve ser feita com cautela em decorrência de algumas limitações. Primeiramente, os dados foram coletados em dois restaurantes distintos. Embora os dois restaurantes possuam similaridades quanto ao públicoalvo, tipo de comida servida e tipo de serviço oferecido aos clientes, as poucas diferenças existentes podem ter influenciado nos resultados. Além disso, os dados foram coletados apenas durante dois domingos consecutivos. Seguindo a orientação de Milliman (1986), um maior número de dias de coleta poderia ter evidenciado uma diferença mais significativa entre as duas condições de reprodução de música analisadas.

Os resultados encontrados também indicam importantes direções para pesquisas futuras. Por exemplo, estudos posteriores poderiam verificar se, de fato, o mecanismo que explica o maior tempo de permanência dos clientes na condição de música ao vivo é a atratividade e o envolvimento gerado por esse tipo de reprodução, já que neste estudo apenas foi proposto ser essa a explicação mais provável. Além disso, poderia ser analisado como a música mecanizada pode ser combinada com outros estímulos atmosféricos intencionalmente manipulados para influenciar o tempo de permanência dos clientes, uma vez que foi verificado que a música reproduzida por dispositivos eletrônicos abre mais espaço para que outras variáveis influenciem o comportamento do consumidor.

\section{REFERÊNCIAS}

BERLYNE, D. E. Aesthetics and psychobiology. 1. ed. New York: [s. n.], 1971.

BISWAS, D. et al. Shining light on atmospherics: how ambient light influences food choices. Journal of Marketing Research, [S. I.], v. 54, n. 1, p. 111-123, 2017.

BISWAS, D.; LUND, K.; SZOCS, C. Sounds like a healthy retail atmospheric strategy: effects of ambient music and background noise on food sales. Journal of the Academy of Marketing Science, [S. I.], v. 47, n. 1, p. 37-55, 2019.

BISWAS, D.; SZOCS, C. The smell of healthy choices: Cross-modal sensory compensation effects of ambient scent on food purchases. Journal of Marketing Research, [S. I.], v. 56, n. 1, p. 123-141, 2019.

CALDWELL, C.; HIBBERT, S. A. The influence of music tempo and musical preference on restaurant patrons' behavior. Psychology \& Marketing, [S. I.], v. 19, n. 11, p. 895-917, 2002.

COSTA, M. F.; FARIAS, S. A. Efeitos da música ao vivo e mecanizada em ambientes de varejo supermercadista. Revista de Administração Contemporânea, Maringá, v. 20, n. 2, p. 154-174, 2016.

DONG, P.; HUANG, X. I.; LABROO, A. A. Cueing morality: the effect of high-pitched music on healthy choice. Journal of Marketing, [S. I.], v. 84, n. 6, 2020.

DONOVAN, R. J.; ROSSITER, J. R. Store atmosphere: an environmental psychology approach. Journal of Retailing, [S. I.], v. 58, n. 1, p. 34-57, 1982.

FREDERICKSON, J. Technology and music performance in the age of mechanical reproduction. International Review of the Aesthetics and Sociology of Music, [S. I.], v. 20, n. 2, p. 193-220, 1989.

FRITH, S. Live Music exchange. Popular Music, [S. I.], v. 32, n. 2, p. 297-301, 2013. 
HARRINGTON, R. J.; OTTENBACHER, M. C.; TREUTER, A. The musicscape model: direct, mediating, and moderating effects in the casual restaurant experience. International Journal of Hospitality \& Tourism Administration, [S. I.], v. 16, n. 2, p. 99-121, 2015.

HERNANDEZ, J. M. C.; BASSO, K.; BRANDÃO, M. M. Pesquisa experimental em marketing. Revista Brasileira de Marketing, [S. I.], v. 13, n. 2, p. 98-117, 2014.

HERSCHMANN, M. Revalorização da música ao vivo em um contexto de crise da indústria fonográfica. In: CONGRESSO BRASILEIRO DE CIÊNCIAS DA COMUNICAÇÃO, 31., 2008. Anais [...]. Natal: Intercom, 2008. p. 1-15.

IAZZETA, F. A Música, o corpo e as máquinas. Revista Opus, [S. I.], v. 4, p. 1-20, 1997.

KOTLER, P. Atmospherics as a marketing tool. Journal of Retailing, [S. I.], v. 49, n. 4, p: 48-65, 1973.

MACIEL, D. F. et al. A influência de estilos musicais no humor, tempo percebido e decisão de retorno em loja de consumo popular. In: ENCONTRO DE MARKETING, 4., 2010. Anais [... ]. Florianópolis: Anpad, 2010. p. 1-17.

MILLIMAN, R. E. The influence of background music on the behavior of restaurant patrons. Journal of Consumer Research, [S. I.], v. 13, n. 2, p. 286-289, 1986.

MILLIMAN, R. E. Using background music to affect the behavior of supermarket shoppers. Journal of Marketing, [S. I.], v. 46, n. 3, p. 86-91, 1982.

SINHA, J.; BAGCHI, R. Role of ambient temperature in influencing willingness to pay in auctions and negotiations. Journal of Marketing, [S. I.], v. 83, n. 4, p. 121-138, 2019.

SMITH, P. C.; CURNOW, R. "Arousal hypothesis" and the effects of music on purchasing behavior. The Journal of Applied Psychology, [S. I.], v. 50, n. 3, p. 255-256, 1966.

SPANGENBERG, E. R.; CROWLEY, A. E.; HENDERSON, P. Improving the store environment: do olfactory cues affect evaluations and behaviours? Journal of Marketing, [S. I.], v. 60, n. 2, p. 67-80, 1996.

SPANGENBERG, E. R.; ROHMANN, B.; SPROTT, D. E. It's beginning to smell (and sound) a lot like Christmas: the interactive effects of ambient scent and music in a retail setting. Journal of Business Research, [S. I.], v. 58, n. 11, 1583-1589, 2005.

SWARBRICK, D. et al. How live music moves us: head movement differences in audiences to live versus recorded music. Frontiers in Psychology, [S. I.], v. 9, p. 2682, 2019.

TURLEY, L. W.; MILLIMAN, R. E. Atmospheric effects on shopping behavior: a review of the experimental evidence. Journal of Business Research, [S. I.], v. 49, n. 2, p. 193-211, 2000.

YALCH, R. F.; SPANGENBERG, E. R. Effects of store music on shopping behavior. Journal of Consumer Marketing, [S. I.], v. 7, n. 2, p. 55-63, 1990.

YALCH, R. F.; SPANGENBERG, E. R. The effects of music in a retail setting on real and perceived shopping times. Journal of Business Research, [S. I.], v. 49, n. 2, p. 139-147, 2000. 


\section{Contato:}

Daniel Max de Sousa Oliveira

E-mail: danielmax2011@gmail.com

\section{Elton Belz}

E-mail: aneltonbelz77@gmail.com

Sylvio Ribeiro de Oliveira Santos

E-mail: sylvio.ros@gmail.com 